\title{
Can test-tube evolution explain biodiversity?
}

Experimental Evolution and the Nature of Biodiversity, by Rees Kassen, Roberts and Company, 2014, [price] ISBN 978-1-936221-46-2

Trends in Ecology and Evolution 30: 568-569.

Tadeusz J. Kawecki

Department of Ecology and Evolution, University of Lausanne, Switzerland

Correspondence: tadeusz.kawecki@unil.ch

What is the distribution of fitness effects of alleles mediating adaptation to a novel environment? How is the evolution of niche breadth affected by environmental variability? How important are antagonistic pleiotropy and epistasis in diversification of lineages? How are rates of diversification affected by ecological interactions? Scientific literature is replete with theories addressing these fundamental questions. However, empirical support for these theories with data from nature is often less than satisfactory, not least because the evolutionary processes that shaped a taxon usually have to be inferred from a single snapshot of its evolutionary history. Enter experimental evolution, which permits direct replicated tests of predictions under controlled conditions [1]. Rees Kassen's Experimental Evolution and the Nature of Biodiversity testifies to the power of experimental evolution in microbial systems to address such questions and foster the development of a general theory of evolutionary adaptation and diversification.

The book is structured by theory. Successive chapters introduce briefly the assumptions, logic and predictions concerning different aspects of adaptation and diversification. Kassen does an excellent job introducing the theory at an intuitive level. This comes at a cost; the theory is often simplified, the diversity of assumptions and predictions are glossed over, and only a few and not always the most relevant theory papers are cited. But a real strength of the book is the thorough review of relevant results from microbial experimental evolution, summarized in extensive tables and correlation plots. Although the book stops short of formal metaanalysis, the evidence 
gathered provides a rather convincing support for some predictions, e.g., that the rate with which successive alleles are substituted during adaptation to a novel environment decreases with time, or that diversification is hindered by the presence of competitors. Question that need more data to be resolved are clearly identified. The focus on general models of adaptation leaves out some more specific topics, such as the evolution of parasite virulence, on which there is both rich theory and substantial body of data from microbial evolution experiments [2]. But within its defined scope it is not only an authoritative review of the evidence, but also a great introduction for nonspecialists to both experimental evolution and the theories of adaptation and diversification.

Although the evidence reviewed in the book is limited to microbial experiments, Kassen's explicit motivation is understanding the nature of biodiversity beyond laboratory and beyond microbes. Jacques Monod famously stated that what is true for $E$. coli is true for an elephant; ironically, his discovery of operons as a major feature of bacterial genome organization turned out not to extrapolate to eukaryotes. Despite carefully discussing limitations and caveats, Kassen might also be too optimistic about the extent to which the results from microbial experimental evolution can be extrapolated to sexual multicellular organisms. First, the book espouses the view that speciation in "macrobes" is usually initiated by ecologically-driven diversifying selection; it plays down the cohesive force of sexual reproduction, implying that reproductive isolation evolves almost as a necessary consequence of the diversifying selection. While such "ecological speciation" does seem to occur [3], the jury is still out as to its importance in generating biodiversity of plants and animals. The alternative view is that reproductive isolation in multicellular sexuals usually arises through accumulation of genetic incompatibilities or through divergence of mate recognition systems by sexual selection, independently of ecological adaptation [4,5]. Thus, ecological diversification may be a consequence rather than the cause of speciation. The data reviewed in this book cannot throw much light on this controversy, and even microbes that engage in occasional sex (e.g., yeast or Chlamydomonas) are not an ideal model system because they lack the extreme asymmetry in gamete size (or investment in offspring) that is the main driver of sexual selection in plants and animals [6]. Second, I am not convinced that the predominance of protein sequence over cis-regulatory changes in microbial evolution experiments helps to resolve the controversy about their relative contribution to diversification of animals and plants [7]. The complex regulatory mechanisms that orchestrate the development and cell 
differentiation of multicellular organisms arguably offer a broader potential target for evolution. Furthermore, adaptation in plants and animals typically involves changes in morphology, physiology or behavior, which are mediated by specific cells and organs rather than by changes in biochemistry of all cells. This leaves more opportunity for evolution to tweak regulatory mechanisms in cell- or tissue-specific way.

A central message of this book does, however, extrapolate beyond microbes: the genetic architecture of adaptive traits does matter for the evolution biodiversity. Thus, a general theory of diversification called for in the last chapter will require a synthesis of ecology and genetics of adaptation (and, for sexual organisms, of reproductive isolation [8]). Such a synthesis is easier in microbes, but this enjoyable book from an expert author motivates one to attempt it in other organisms.

\section{References}

1. Kawecki, T.J., et al. (2012) Experimental evolution. Trends Ecol. Evol. 27, 547-560.

2. Alizon, S., et al. (2009) Virulence evolution and the trade-off hypothesis: history, current state of affairs and the future. J. Evol. Biol. 22, 245-259.

3. Schluter, D. and Conte, G.L. (2009) Genetics and ecological speciation. Proc. Natl. Acad. Sci. USA 106, 9955-9962.

4. Coyne, J.A. and Orr, H.A. (2004) Speciation. Sinauer Associates.

5. Butlin, R., et al. (2012) What do we need to know about speciation? Trends Ecol. Evol. 27, 27-39.

6. Schärer, L., et al. (2012) Anisogamy, chance and the evolution of sex roles. Trends Ecol. Evol. 27, 260-264.

7. Hoekstra, H.E. and Coyne, J.A. (2007) The locus of evolution: Evo devo and the genetics of adaptation. Evolution 61, 995-1016.

8. Nosil, P. and Schluter, D. (2011) The genes underlying the process of speciation. Trends Ecol. Evol. 26, 160-167. 